\title{
Structure Activity Relationships, QSAR Modeling and Drug-like calculations of TP inhibition of 1,3,4- oxadiazoline-2-thione Derivatives
}

\author{
Zineb Almi ${ }^{1}$, Salah Belaidi ${ }^{1, *}$, Touhami Lanez ${ }^{2}$, Noureddine Tchouar $^{3}$ \\ ${ }^{1}$ Group of Computational and Pharmaceutical Chemistry, LCME Laboratory, Department of Chemistry, \\ Faculty of Sciences, University of Biskra, 07000, Biskra, Algeria \\ ${ }^{2}$ VTRS Laboratory, Faculty of Sciences and Technology, University of El-Oued, \\ B.P.789, 39000, El-Oued, Algeria \\ ${ }^{3}$ Dept. of Chemistry Physics, LAMOSI Laboratory, Faculty of Chemistry, University of USTO-MB, \\ BP 1503 Oran 31000, Algeria \\ *E-mail address: prof.belaidi @laposte.net
}

\begin{abstract}
QSAR studies have been performed on twenty-one molecules of 1,3,4-oxadiazoline-2-thiones. The compounds used are among the most thymidine phosphorylase (TP) inhibitors. A multiple linear regression (MLR) procedure was used to design the relationships between molecular descriptor and TP inhibition of the 1,3,4-oxadiazoline-2-thione derivatives. The predictivity of the model was estimated by cross-validation with the leave-one-out method. Our results suggest a QSAR model based of the following descriptors: $\log$ P, HE, Pol, MR, MV, and MW, qO1, SAG, for the TP inhibitory activity. To confirm the predictive power of the models, an external set of molecules was used. High correlation between experimental and predicted activity values was observed, indicating the validation and the good quality of the derived QSAR models.
\end{abstract}

Keywords: SAR; QSAR; Drug-like; TP inhibitory; MLR; 1,3,4-oxadiazoline-2-thione derivatives

\section{INTRODUCTION}

QSAR, a quantum chemical technique is known to relate the biological activity of compounds with their molecular structure and has been extensively used as predicting tool in rational drug design [1]. Quantitative structure - activity relationships (QSARs), as one of the most important areas in chemometrics, QSAR models are mathematical equations relating chemical structure to their biological activity. QSAR are attempts to correlate molecular structure, or properties derived from molecular structure with a particular kind of chemical or biochemical activity [2].

Multiple linear regression (MLR) is also a mathematical tool that quantifies the relationship between a dependent variable and one or more independent variables. Thus, angiogenesis inhibitors are believed to be potential candidates for blocking cancer growth. In particular, thymidine phosphorylase (TP) is a pro-angiogenic factor which catalyzes the reversible phosphorolysis of thymidine into thymine and 2'-deoxy-D-ribose 1-phosphate [3]. 
TP inhibitors affect the production of 2-deoxy-D-ribose and in turn suppress tumor growth. Therefore, there is an urgent need to develop new and potent thymidine phosphorylase inhibitors which have the ability to suppress the formation of new blood vessels and stop tumor growth. A number of efforts have been reported on the development of TP inhibitors [4].

Drug-likeness is a qualitative concept used in drug design, which is estimated from the molecular structure before the substance is even synthesized and tested. The calculation of drug-like property can give us better assumption of biological activity of certain molecule. The theoretical calculation of certain properties of a molecule can fill the parameters, which are essential to show certain biological activity. Lipinski's rule of five (ROF) is a rule of thumb to evaluate drug-likeness or determine a chemical compound with a certain pharmacological or biological activity that would make it a likely orally active drug in humans [5].

The ROF is based on four properties of molecules, namely, molecular weight (MW), $\log$, number of hydrogen-bond donors (HBD) taken as equivalent to the number of - $\mathrm{OH}$ and -NH groups, and the number of hydrogen-bond acceptors (HBA) taken as equivalent to the number of oxygen and nitrogen atoms.

A 'flag' is set if a molecule's MW is greater than 500, its $\log \mathrm{P}$ is greater than 5 , the number of its HBDs exceeds 5 and the number of its HBAs exceeds 10. Because the values of the decision points for all of the property values are multiples of five, the above set of rules has been called the 'Rule of Five.' The total number of violations is the ROF-Score, which lies between ' 0 ' and ' 4 ' [6].

Following our interest in this field, our present research aimed to describe the structureproperty relationships study on 1,3,4-oxadiazoline-2-thiones and developed a QSAR model on these compounds with respect to their TP inhibitory activity (TPI).

\section{EXPERIMENTAL}

\section{1. Biological data}

The activity parameter used in this study is thymidine phosphorylase (TP) inhibitory activity. The studied compounds are TP inhibitors which inhibit tumor growth. Interestingly, all these compounds were active and showed TP inhibition with IC50 values ranged between $14.40 \pm 2.45$ and $173.23 \pm 3.04 \mu \mathrm{M}[7]$.

\section{2. Descriptors generation}

Firstly, the twenty-one investigated molecules were pre-optimized by means of the Molecular Mechanics Force Field (MM+) included in HyperChem version 8.03 package [8] . After that, the resulted minimized structures were further refined using the semi-empirical PM3 Hamiltonian implemented also in HyperChem. We chose a gradient norm limit of $0.01 \mathrm{kcal} / \AA$ for the geometry optimization. Then, these $1,3,4$-oxadiazoline-2-thiones were reoptimized by using Gaussian 09 program package [9], at the density functional theory level DFT using Becke's three-parameter Lee-Yang- Parr (B3LYP), with the 6-311G d, p basis set, this theory was used to calculate a number of electronic descriptors: dipole moment (DM), energy of frontier orbital's, EHOMO and ELUMO, and atomic net charges (qO1, qC2,qN3,qN4,qC5,qS6).

The QSAR properties module from HyperChem 8.03 was used to calculate: molar polarizability (Pol), the molar refractivity (MR), partition coefficient octanol/water $(\log \mathrm{P})$, 
hydration energy (HE), molar volume (MV), Surface area grid (SAG) and molar weight (MW).

Calculation of $\log \mathrm{P}$ is carried out using atomic parameters derived by Viswanadhan and coworkers [10]. Computation of molar refractivity was made via the same method as $\log P$. Ghose and Crippen presented atomic contributions to the refractivity [11]. Solventaccessible surface bounded molecular volume and van der Waals-surface-bounded molecular volume calculations are based on a grid method derived by Bodor et al., [12] using the atomic radii of Gavezotti [13]. Polarizability was estimated from additivity scheme given by Miller with a $3 \%$ in precision for the calculation [14], where different increments are associated with different atom types.

\section{3. Regression analysis}

Multiple linear regression analysis of molecular descriptors was carried out using the stepwise strategy in SPSS version 19 for Windows [15].

\section{RESULTS AND DISCUSSION}

\section{1. Structure activity relationships (SAR)}

We have studied seven physical chemical proprieties of series of twenty-one 1,3,4oxadiazoline-2-thione derivatives (3a-3u) in which, various degrees of substituents on phenyl have been introduced, these substituents include electron donating group such as methoxy and electron withdrawing group like nitro [7], using HyperChem software. QSAR proprieties such as van der Waals surface molecular volume, octanol-water partition coefficient (log P), molar refractivity (MR), polarizability (Pol), solvent-accessible, surface bounded molecular volume and molecular weight $(\mathrm{M})$ were investigated

Table 1. Chemical structures and experimental activity of the 1,3,4-oxadiazoline-2-thione derivatives $3 a-3 u$.

\begin{tabular}{|c|c|c|c|c|c|c|}
\hline \multicolumn{2}{|c|}{ Compound } & $\mathrm{R}_{1}$ & $\mathrm{R}_{2}$ & $\mathrm{P}_{\text {IC50 }}$ exp & $\mathrm{P}_{\text {IC50 }}$ pred & Residu \\
\hline 1 & $3 \mathrm{a}$ & Phenyl & 2-Methoxyphenyl & 4.059 & 4.177 & -0.118 \\
\hline 2 & $3 \mathrm{~b}$ & Phenyl & 3-Methoxyphenyl & 4.391 & 4.408 & -0.017 \\
\hline 3 & $3 \mathrm{c}$ & Phenyl & 2-Chlorophenyl & 3.929 & 4.199 & -0.270 \\
\hline 4 & $3 \mathrm{~d}$ & Phenyl & 3,4-Dimethylphenyl & 4.391 & 4.170 & 0.221 \\
\hline 5 & $3 \mathrm{e}$ & $\begin{array}{c}\text { 3,4,5- } \\
\text { Trimethoxyphenyl }\end{array}$ & 2-Methylphenyl & 3.931 & 4.023 & -0.092 \\
\hline 6 & $3 \mathrm{f}$ & $\begin{array}{c}\text { 3,4,5- } \\
\text { Trimethoxyphenyl }\end{array}$ & 2-Ethylphenyl & 4.159 & 4.219 & -0.060 \\
\hline 7 & $3 \mathrm{~g}$ & 2-Hydroxyphenyl & 2-Chlorophenyl & 3.884 & 3.829 & 0.054 \\
\hline 8 & $3 \mathrm{~h}$ & 4-Methylphenyl & $\begin{array}{c}\text { 2-Methoxy-5- } \\
\text { nitrophenyl }\end{array}$ & 4.842 & 4.531 & 0.311 \\
\hline 9 & $3 \mathrm{i}$ & 4-Methylphenyl & 4-Methylphenyl & 4.262 & 4.009 & 0.253 \\
\hline 10 & $3 \mathrm{j}$ & 4-Methylphenyl & Phenyl & 3.982 & 4.009 & -0.028 \\
\hline
\end{tabular}




\begin{tabular}{|c|c|c|c|c|c|c|}
\hline 11 & $3 \mathrm{k}$ & 4-Methylphenyl & 2,3-Dimethylphenyl & 4.625 & 4.447 & 0.178 \\
\hline 12 & 31 & 4-Methylphenyl & 3,4-Dichlorophenyl & 3.874 & 3.996 & -0.122 \\
\hline 13 & $3 \mathrm{~m}$ & 4-Methylphenyl & 3,4-Dimethylphenyl & 4.080 & 4.231 & -0.151 \\
\hline 14 & $3 \mathrm{n}$ & 4-Methylphenyl & 3-Methoxyphenyl & 4.074 & 4.180 & -0.106 \\
\hline 15 & $3 \mathrm{o}$ & 2-Bromophenyl & 2-Chlorophenyl & 4.661 & 4.417 & 0.244 \\
\hline 16 & $3 \mathrm{p}$ & 2-Bromophenyl & 4-Bromophenyl & 4.579 & 4.596 & -0.018 \\
\hline 17 & $3 \mathrm{q}$ & 2-Bromopheny & 2-Methoxyphenyl & 4.754 & 4.843 & -0.089 \\
\hline 18 & $3 \mathrm{r}$ & 4-Chlorophenyl & 2-Methylphenyl & 4.235 & 4.246 & -0.011 \\
\hline 19 & $3 \mathrm{~s}$ & 4-Chlorophenyl & 4-Bromophenyl & 3.761 & 3.830 & -0.069 \\
\hline 20 & $3 \mathrm{t}$ & 4-Chlorophenyl & 2-Chlorophenyl & 4.334 & 4.179 & 0.155 \\
\hline 21 & $3 \mathrm{u}$ & 4-Chlorophenyl & 2-Methoxyphenyl & 4.412 & 4.674 & -0.262 \\
\hline
\end{tabular}

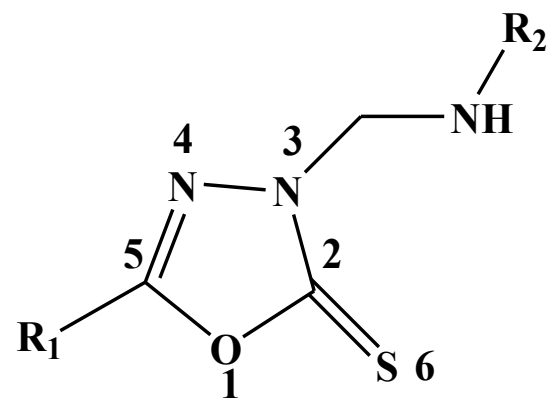

Molecular polarizability of a molecule characterizes the capability of its electronic system to be distorted by the external field, and it plays an important role in modeling many molecular properties and biological activities [16,21-24]. The attractive part of the Van der Waals interaction is a good measure of the polarizability. Highly polarizable molecules can be expected to have strong attractions with other molecules. The polarizability of a molecule can also enhance aqueous solubility. The molar refractivity (MR) is important criterion to measure the steric factor. It is usually designated as a simple measure of the volume occupied either by an individual atom or a cluster (group) of atoms [17]. Polarizability and molar refractivity relatively increase with the size and the molecular weight of the studied phenothiazines (Table 2). This result is in agreement with the formula of Lorentz-Lorenz which gives a relationship between polarizability, the molar refractivity and volume [18]. This relationship shows that the polarizability and the molar refractivity increase with the volume and the molecular weight. For example, the compound 6 has great values of polarizability (43.43) and molar refractivity (118.54). In contrast, the compound 10 is the small molecule in the series of studied 1,3,4-oxadiazoline-2-thiones, which has a small value of polarizability (34.18), and of molar refractivity (94.82) . 
Table 2. Values of molecular descriptors used in the regression analysis.

\begin{tabular}{|c|c|c|c|c|c|c|c|c|c|c|}
\hline Compound & $\mathrm{E}_{\text {НОмо }}$ & $\mathrm{E}_{\text {LUMO }}$ & $\mathrm{DM}$ & $\log P$ & $\mathrm{HE}$ & Pol & MR & MV & SAG & MW \\
\hline 1 & -0.218 & -0.068 & 5.434 & 1.70 & -9.04 & 34.82 & 96.91 & 898.24 & 537.96 & 313.37 \\
\hline 2 & -0.206 & -0.073 & 4.782 & 1.70 & -9.04 & 34.82 & 96.91 & 876.92 & 511.52 & 313.37 \\
\hline 3 & -0.229 & -0.069 & 2.629 & 2.47 & -7.80 & 34.27 & 95.25 & 857.96 & 505.74 & 317.79 \\
\hline 4 & -0.205 & -0.070 & 4.430 & 3.00 & -6.50 & 36.01 & 99.10 & 905.51 & 521.91 & 311.40 \\
\hline 5 & -0.209 & -0.062 & 6.153 & -0.14 & -10.15 & 41.60 & 113.94 & 1081.62 & 616.64 & 387.45 \\
\hline 6 & -0.209 & -0.062 & 6.142 & 0.26 & -9.61 & 43.43 & 118.54 & 1126.64 & 642.74 & 401.48 \\
\hline 7 & -0.217 & -0.068 & 6.617 & 1.44 & -11.18 & 34.91 & 96.86 & 879.25 & 521.86 & 333.79 \\
\hline 8 & -0.222 & -0.085 & 1.856 & -0.90 & -11.24 & 38.49 & 106.91 & 999.19 & 578.43 & 372.40 \\
\hline 9 & -0.207 & -0.066 & 4.700 & 3.00 & -6.23 & 36.01 & 99.10 & 925.45 & 544.21 & 311.40 \\
\hline 10 & -0.206 & -0.066 & 4.900 & 2.84 & -7.46 & 34.18 & 94.82 & 877.66 & 523.61 & 297.37 \\
\hline 11 & -0.208 & -0.066 & 4.160 & 3.15 & -5.35 & 37.85 & 103.38 & 959.11 & 563.55 & 325.43 \\
\hline 12 & -0.225 & -0.071 & 3.495 & 2.40 & -6.78 & 38.03 & 104.25 & 954.57 & 558.98 & 366.26 \\
\hline 13 & -0.208 & -0.066 & 4.160 & 3.15 & -5.32 & 37.85 & 103.38 & 956.43 & 549.62 & 325.43 \\
\hline 14 & -0.205 & -0.069 & 5.419 & 1.85 & -8.71 & 36.65 & 101.19 & 925.98 & 536.13 & 327.40 \\
\hline 15 & -0.232 & -0.076 & 1.856 & 2.52 & -7.22 & 36.90 & 102.79 & 893.93 & 516.23 & 396.69 \\
\hline 16 & -0.218 & -0.076 & 4.948 & 2.79 & -7.28 & 37.60 & 105.60 & 934.96 & 549.24 & 441.14 \\
\hline 17 & -0.204 & -0.074 & 4.626 & 1.75 & -7.24 & 37.44 & 104.44 & 920.29 & 523.23 & 392.27 \\
\hline 18 & -0.229 & -0.079 & 3.054 & 2.62 & -6.95 & 36.11 & 99.53 & 903.23 & 530.24 & 331.82 \\
\hline 19 & -0.221 & -0.082 & 2.763 & 2.52 & -7.97 & 36.90 & 102.79 & 928.84 & 554.29 & 396.69 \\
\hline 20 & -0.233 & -0.078 & 2.372 & 2.24 & -7.45 & 36.20 & 99.97 & 900.24 & 529.59 & 352.24 \\
\hline 21 & -0.211 & -0.076 & 4.446 & 1.47 & -7.47 & 36.74 & 101.63 & 923.44 & 536.46 & 347.82 \\
\hline
\end{tabular}

$\mathrm{E}_{\mathrm{HOMO}}, \mathrm{E}_{\mathrm{LUMO}}, \mathrm{DM}$ calculated by DFT/6-311G d,p (Gaussian09)

Table 2. Continued.

\begin{tabular}{|c|c|c|c|c|c|c|}
\hline Compound & $\mathrm{q} 01$ & $\mathrm{qC} 2$ & $\mathrm{qN} 3$ & $\mathrm{qN} 4$ & $\mathrm{qC} 5$ & $\mathrm{qS} 6$ \\
\hline 1 & -0.262 & 0.189 & -0.302 & -0.230 & 0.423 & -0.182 \\
\hline 2 & -0.260 & 0.205 & -0.285 & -0.213 & 0.417 & -0.206 \\
\hline 3 & -0.259 & 0.198 & -0.281 & -0.193 & 0.412 & -0.208 \\
\hline 4 & -0.260 & 0.205 & -0.285 & -0.208 & 0.415 & -0.211 \\
\hline
\end{tabular}




\begin{tabular}{|c|c|c|c|c|c|c|}
\hline 5 & -0.258 & 0.207 & -0.283 & -0.221 & 0.425 & -0.216 \\
\hline 6 & -0.257 & 0.208 & -0.282 & -0.222 & 0.426 & -0.217 \\
\hline 7 & -0.233 & 0.199 & -0.282 & -0.217 & 0.426 & -0.207 \\
\hline 8 & -0.259 & 0.201 & -0.284 & -0.212 & 0.409 & -0.211 \\
\hline 9 & -0.261 & 0.206 & -0.285 & -0.210 & 0.415 & -0.216 \\
\hline 10 & -0.259 & 0.202 & -0.282 & -0.210 & 0.407 & -0.209 \\
\hline 11 & -0.260 & 0.207 & -0.283 & -0.211 & 0.417 & -0.216 \\
\hline 12 & -0.259 & 0.205 & -0.285 & -0.213 & 0.418 & -0.209 \\
\hline 13 & -0.260 & 0.204 & -0.285 & -0.208 & 0.419 & -0.217 \\
\hline 14 & -0.260 & 0.206 & -0.285 & -0.215 & 0.416 & -0.211 \\
\hline 15 & -0.266 & 0.202 & -0.278 & -0.185 & 0.414 & -0.206 \\
\hline 16 & -0.232 & 0.201 & -0.284 & -0.193 & 0.313 & -0.199 \\
\hline 17 & -0.265 & 0.207 & -0.282 & -0.180 & 0.387 & -0.215 \\
\hline 18 & -0.261 & 0.195 & -0.282 & -0.201 & 0.417 & -0.196 \\
\hline 19 & -0.260 & 0.203 & -0.285 & -0.209 & 0.421 & -0.198 \\
\hline 20 & -0.260 & 0.198 & -0.281 & -0.192 & 0.416 & -0.202 \\
\hline 21 & -0.262 & 0.196 & -0.282 & -0.195 & 0.414 & -0.204 \\
\hline
\end{tabular}

Net charge calculated by DFT/6-311G d,p (Gaussian09)

The presence of the hydrophobic groups in the structure of the 1,3,4-oxadiazoline-2thiones induces a decrease of the hydratation energy, however, the presence of hydrophilic groups increases the hydratation energy (Table 2).

The most important hydratation energy in the absolute value, $(11.24 \mathrm{kcal} / \mathrm{mol})$ is that of the compound 8 , but the lower one $(5.32 \mathrm{kcal} / \mathrm{mol})$ was performed for the compound 13 (Table 2). Indeed in the biological environment the polar molecules are surrounded by water molecules where the Hydrogen bonds can be established between the water molecule and the molecules under study. The donor sites of proton interact with the oxygen atom of water and the acceptor sites of proton interact with the hydrogen atom. The first corresponds to the complex having strongest hydrogen bond. At least, these hydrated molecules are partially dehydrated before their interaction. These interactions of weak energy are generally reversible in particular between messengers and receivers.

Compound 8 has one donor site of proton $(1 \mathrm{NH})$, but it has nine acceptor sites of proton (4N and $4 \mathrm{O}, 1 \mathrm{~S})$. On the other hand, the compound 13 has one donor site of proton $(1 \mathrm{NH})$, but it has five acceptor sites of proton $(3 \mathrm{~N}, 1 \mathrm{O}, 1 \mathrm{~S})$. The first having higher value, it has four more acceptor sites of proton. This property supports the compound 8 not only by fixing the receptors, but also activates it by playing the role of agonist. It has as a consequence a better distribution in fabrics [19].

Lipophilicity is a property that has a major effect on solubility, absorption, distribution, metabolism, and excretion properties as well as pharmacological activity. Hansch and Leo reasoned that highly lipophilic molecules will partition into the lipid interior of membranes 
and retained there. For good oral bioavailability, $\log \mathrm{P}$ must be in the range $(0<\log \mathrm{P}<3)$. For higher $\log \mathrm{P}$ the drug has low solubility and for lower $\log \mathrm{P}$, the drug has difficulty to penetrate the lipid membranes [3]. In opposition to hydratation energy, the presence of the hydrophobic groups in the structure of the 1,3,4-oxadiazoline-2-thiones induces an increase of the lipophilicity. Compound 8 presents the low coefficient of division $(-0.90)$. When the coefficient of division is rather low, it has as a consequence a better gastric tolerance. Compounds 11 and 13 which have higher value (3.15), have capacities to be dependent on plasmatic proteins.

\section{2. Quantitative structure-activity relationships studies}

Firstly, different substituted 1,3,4-oxadiazoline-2-thiones (Table 1) were evaluated for their TP inhibitory activity. The biological parameter (IC50) was introduced in this search and the results are illustrated in Table 1. In order to determine the role of structural features. A series of twenty-one 1,3,4-oxadiazoline-2-thiones was investigated by QSAR method.

These compounds were used for multilinear regression model generation. Different physicochemical descriptors such as steric, electronic and molecular structure were used as independent variables and were correlated with biological activity.

Developing a QSAR model requires a diverse set of data, and, thereby a large number of descriptors have to be considered. Descriptors are numerical values that encode different structural features of the molecules.

Selection of a set of appropriate descriptors from a large number of them requires a method, which is able to discriminate between the parameters. Pearson's correlation matrix has been performed on all descriptors by using SPSS Software. The analysis of the matrix revealed sixteen descriptors for the development of MLR model. The values of descriptors selected for MLR model are presented in Table 2.

The correlation between the biological activity (IC50) and descriptors expressed by the following relation:

$$
\begin{gathered}
\mathrm{PIC} 50=3.028-0.542 \log \mathrm{P}+0.352 \mathrm{HE}-1.272 \mathrm{Pol}+0.863 \mathrm{MR}-0.038 \mathrm{MV}-0.024 \mathrm{MW}+ \\
19.120 \mathrm{qO} 1+0.024 \mathrm{SAG} . \\
n=21 ; r=0.848 ; s=0.216 ; F=3.844 ; Q=3.926
\end{gathered}
$$

The values of fraction variance may vary between 0 and 1 . QSAR model having $\mathrm{r}^{2}>0.6$ will only be considered for validation. For example, the value $r=0.848$ and $r^{2}=0.719$ allowed us to indicate firmly the correlation between different parameters (independent variables) with TP inhibition of the compounds.

The F-value has found to be statistically significant at $95 \%$ level, since the calculated $\mathrm{F}$ value is higher as compared to tabulated value. The positive value of quality factor (Q) for this QSAR's model suggests its high predictive power and lack of over fitting.

In equation of PIC50, the negative coefficients of MV and MW explain that any increase in molecular volume or molecular weight of the compounds causes a decrease in the biological activity.

In order to test the validity of the predictive power of selected MLR model (eq. PIC50), the leave-one-out technique (LOO technique) was used. The developed models were validated by calculation of the following statistical parameters: predicted residual sum of squares (PRESS), total sum of squares deviation (SSY) and cross-validated correlation coefficient ( $\mathrm{r}^{2}$ adj) (Table 3$)$. 
Table 3. Cross-validation parameters.

\begin{tabular}{|c|c|c|c|c|c|c|}
\hline Model & PRESS & SSY & PRESS/SSY & S $_{\text {PRESS }}$ & $\mathrm{r}^{2}{ }_{\mathrm{cv}}$ & $\mathrm{r}_{\text {adj }}^{2}$ \\
\hline PIC50 & 0.559 & 1.991 & 0.280 & 0.163 & 0.719 & 0.532 \\
\hline
\end{tabular}

PRESS is an important cross-validation parameter as it is a good approximation of the real predictive error of the model. Its value being less than SSY points out that model predicts better than chance and can be considered statically significant. The smaller PRESS value means the better of the model predictability. From the results depicted in Table 3, the model is statistically significant.

Also, for reasonable QSAR model, the PREES/SSY ratio should be lower than 0.4 [3]. The data presented in Table 3 indicate that for the developed model this ratio is 0.280 . Our result of $r^{2} c v$ for this QSAR model has been to be 0.719 . The high value of $r^{2} c v$ and $r^{2}$ adj are essential criteria for the best qualification of the QSAR model.

However, the only way to estimate the true predictive power of developed model is to predict the by calculation of PIC50 values of the investigated 1,3,4-oxadiazoline-2-thiones using this model (Table 1).

Figure 1 shows the plots of linear regression predicted versus experimental value of the biological activity of 1,3,4-oxadiazoline-2-thiones outlined above. The plots for this model show to be more convenient with $r^{2}=0.719$. It indicates that the model can be successfully applied to predict the TP inhibitory activity of these compounds.

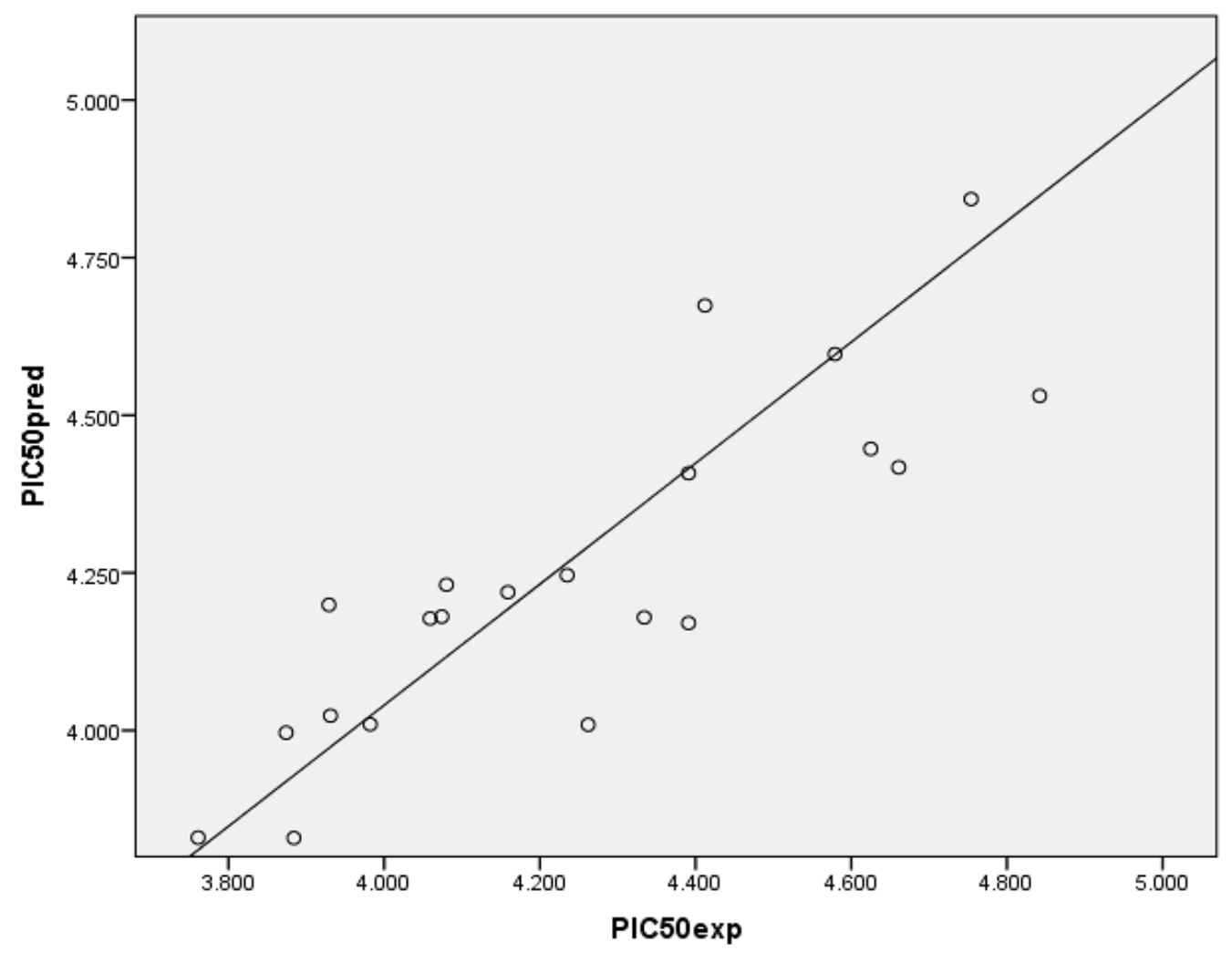

Fig. 1. Predicted plot versus experimental observed TP inhibition of 1,3,4-oxadiazoline-2-thiones. 


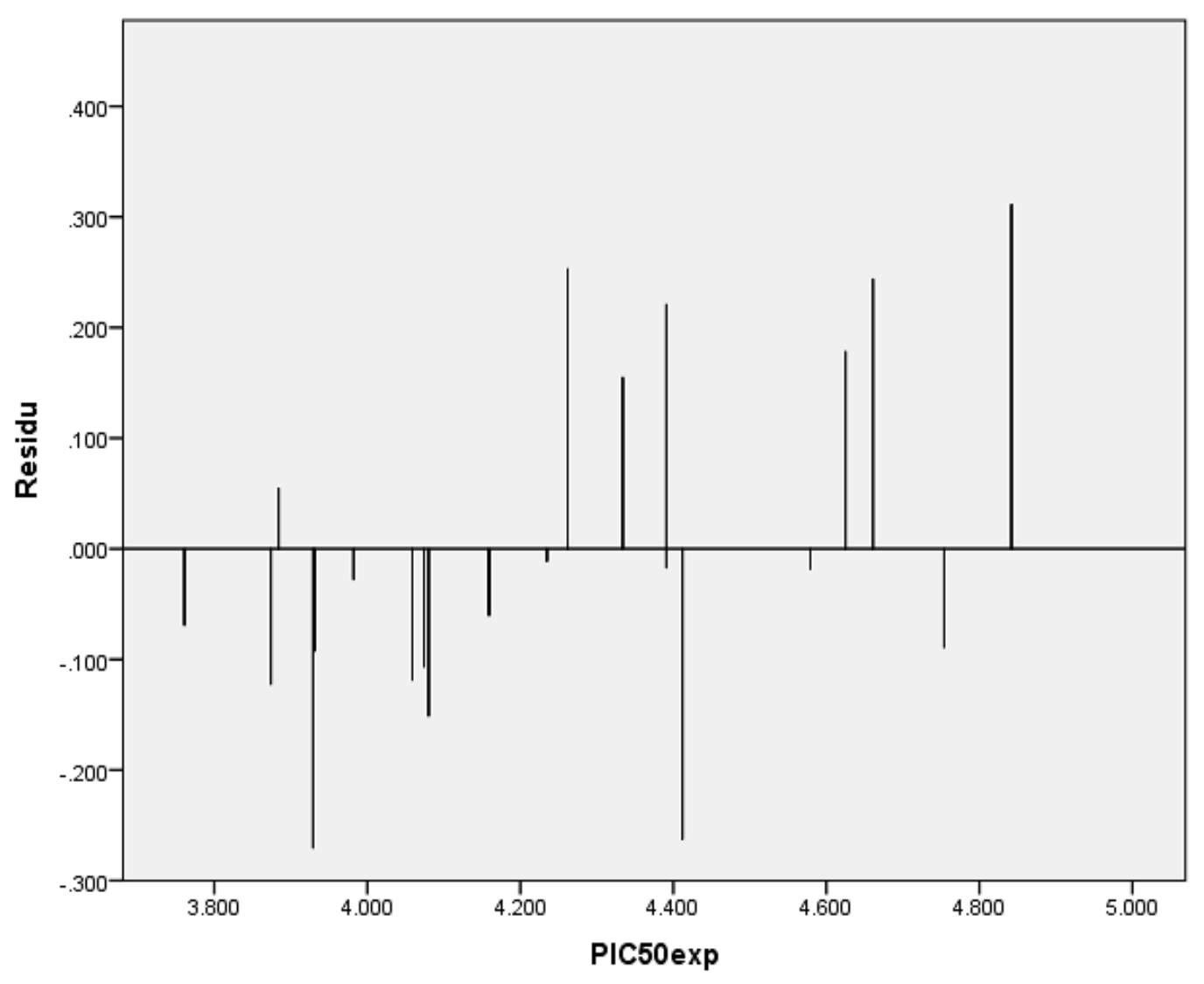

Fig. 2. Plot of the residual values against the experimentally observed (PIC50).

\section{3. Drug-like calculation on the basis of Lipinski rule of five}

Drug-like appears as a promising paradigm to encode the balance among the molecular properties of a compound that influences its pharmacodynamics and pharmacokinetics and ultimately optimizes their absorption, distribution, metabolism and excretion (ADME) in human body like a drug. The empirical conditions to satisfy Lipinski's rule and manifest a good oral bioavailability involve a balance between the aqueous solubility of a compound and its ability to diffuse passively through the different biological barriers [8]. These parameters allow ascertaining oral absorption or membrane permeability that occurs when the evaluated molecule follows Lipinski's rule of five since molecular weight (MW) $\leq 500 \mathrm{Da}$, an octanolwater partition coefficient $\log \mathrm{P} \leq 5, \mathrm{H}$-bond donors, nitrogen or oxygen atoms with one or more hydrogen atoms (HBD) $\leq 5$ and H-bond acceptors, nitrogen or oxygen atoms (HBA) $\leq$ 10.

Molecules that violate more than one of these rules may have problems with bioavailability. Therefore, this rule establishes some structural parameters relevant to the theoretical prediction of the oral bioavailability profile, and is widely used in designing new drugs. However, classes of compounds that are substrates for biological transporters such as antibiotics, antifungals, vitamins, and cardiac glycosides, are exceptions to the rule. The total number of violations is the ROF-Score, which lies between 0 and 4 [20].

The calculation results (Table 4) show that all the studied compounds agree with Lipinski rules with ROF-Score $\leq 1$, suggesting that these compounds theoretically would not have problems with oral bioavailability. Molecules with ROF-Scores greater than one are 
considered to be marginal for further development. Although, as pointed out by Lipinski and co-workers. Lastly, it is well known that many drugs violate the ROF, but this is not a serious issue since it was not originally designed as a tool for assessing drug likeness. Nevertheless, its common usage for this purpose has, de facto, made it so in practice.

Table 4. Lipinski's rule of five for drug likeliness of 1,3,4-oxadiazoline-2-thione derivatives

\begin{tabular}{|c|c|c|c|c|c|}
\hline $\begin{array}{c}1,3,4- \\
\text { oxadiazoline-2- } \\
\text { thione } \\
\text { derivatives } \\
\end{array}$ & $\begin{array}{l}\text { Molecular } \\
\text { Mass (uma) }\end{array}$ & $\log \mathrm{P}$ & HBD & HBA & $\begin{array}{l}\text { violations of } \\
\text { Lipinski rule }\end{array}$ \\
\hline 1 & 313.37 & 1.70 & 1 & 5 & 0 \\
\hline 2 & 313.37 & 1.70 & 1 & 5 & 0 \\
\hline 3 & 317.79 & 2.47 & 1 & 4 & 0 \\
\hline 4 & 311.40 & 3.00 & 1 & 4 & 0 \\
\hline 5 & 387.45 & -0.14 & 1 & 7 & 0 \\
\hline 6 & 401.48 & 0.26 & 1 & 7 & 0 \\
\hline 7 & 333.79 & 1.44 & 2 & 5 & 0 \\
\hline 8 & 372.40 & -0.90 & 1 & 8 & 0 \\
\hline 9 & 311.40 & 3.00 & 1 & 4 & 0 \\
\hline 10 & 297.37 & 2.84 & 1 & 4 & 0 \\
\hline 11 & 325.43 & 3.15 & 1 & 4 & 0 \\
\hline 12 & 366.26 & 2.40 & 1 & 4 & 0 \\
\hline 13 & 325.43 & 3.15 & 1 & 4 & 0 \\
\hline 14 & 327.40 & 1.85 & 1 & 5 & 0 \\
\hline 15 & 396.69 & 2.52 & 1 & 4 & 0 \\
\hline 16 & 441.14 & 2.79 & 1 & 4 & 0 \\
\hline 17 & 392.27 & 1.75 & 1 & 5 & 0 \\
\hline 18 & 331.82 & 2.62 & 1 & 4 & 0 \\
\hline 19 & 396.69 & 2.52 & 1 & 4 & 0 \\
\hline 20 & 352.24 & 2.24 & 1 & 4 & 0 \\
\hline 21 & 347.82 & 1.47 & 1 & 5 & 0 \\
\hline
\end{tabular}

MM and Log P calculated by HyperChem 8.06

\section{CONCLUSIONS}

Based on the present investigation it can be concluded that the model " PIC50 $=3.028$ $0.542 \log \mathrm{P}+0.352 \mathrm{HE}-1.272 \mathrm{Pol}+0.863 \mathrm{MR}-0.038 \mathrm{MV}-0.024 \mathrm{MW}+19.120 \mathrm{qO} 1+$ 
0.024SAG" can be useful for predicting the activity of new 1,3,4-oxadiazoline-2-thione derivatives prior to their synthesis. LogP, HE, Pol, MR, MV, and MW, qO1, SAG, are reliable descriptor for predicting activity. QSAR model indicates that these descriptors have significant relationships with observed bioactivity. We have observed a high relationship between experimental and predicted activity values, indicating the validation and the excellent quality of the derived QSAR model.

The application of Lipinski rules on the studied 1,3,4-oxadiazoline-2-thione derivatives shows that all these compounds, theoretically, will not have problems with oral bioavailability.

\section{References}

[1] M. J. S. Dewar, E. G. Zoebisch, E. F. Healy, J.J.P. Stewart, J. Am. Chem. Soc. 107 (1985)

[2] N. Melkemi, S. Belaidi, J. Comput. Theor. Nanosci. 11 (2014) 801-806.

[3] V. A. McNally, M. Rajabi, A. Gbaj, I. J.Stratford, P. N. Edwards, K. T. Douglas, R. A. Bryce, M. Jaffar, S. Freeman, J. Pharm. Pharmacol. 59 ( 2007) 537

[4] P.A., Schwartz, M.J., Vetticatt, V.L., Schramm, Biochemistry 50 (2011) 1412.

[5] M. Joydeep, Ch. Raja, S. Saikat, V. anjay, De. Biplab, T. K. Ravi, Der. Pharma. Chemica 1(2) (2009) 188-198.

[6] P. Joachim, M. Nathalie, K. Christine, M. Gerald, Bioorg \& Med. Chemistry 20 (2012) 5343-5351.

[7] S. A. Shahzad. M. Yar, M. Bajda, B. Jadoon, Z. Ali Khan, S. A. Raza Naqvi, A. J. Shaikh, K. Hayat, A. Mahmmod, N. Mahmood, S. Filipek. Bioorg. Med. Chem. 22 (2014) 1008-1015.

[8] Hyperchem (Molecular Modeling System) Hypercube, Inc., 1115 Nw, 4th Street, Gainesville, Fl 32601; Usa, (2007).

[9] Gaussian 09, Revision B.01, M. J. Frisch, G. W. Trucks, H. B. Schlegel, G. E. Scuseria, M. A. Robb, J. R. Cheeseman, G. Scalmani, V. Barone, B. Mennucci, G. A. Petersson, H. Nakatsuji, M. Caricato, X. Li, H. P. Hratchian, A. F. Izmaylov, J. Bloino, G. Zheng, J. L. Sonnenberg, M. Hada, M. Ehara, K. Toyota, R. Fukuda, J. Hasegawa, M. Ishida, T. Nakajima, Y. Honda, O. Kitao, H. Nakai, T. Vreven, J. A. Montgomery, Jr., J. E. Peralta, F. Ogliaro, M. Bearpark, J. J. Heyd, E. Brothers, K. N. Kudin, V. N. Staroverov, T. Keith, R. Kobayashi, J. Normand, K. Raghavachari, A. Rendell, J. C. Burant, S. S. Iyengar, J. Tomasi, M. Cossi, N. Rega, J. M. Millam, M. Klene, J. E. Knox, J. B. Cross, V. Bakken, C. Adamo, J. Jaramillo, R. Gomperts, R. E. Stratmann, O. Yazyev, A. J. Austin, R. Cammi, C. Pomelli, J. W. Ochterski, R. L. Martin, K. Morokuma, V. G. Zakrzewski, G. A. Voth, P. Salvador, J. J. Dannenberg, S. Dapprich, A. D. Daniels, O. Farkas, J. B. Foresman, J. V. Ortiz, J.Cioslowski and D. J. Fox, Gaussian, Inc., Wallingford CT, (2010).

[10] V. N. Viswanadhan, A. K. Ghose, G. R. Revankar, And R. K. Robins, J. Chem. Inf. Comp. Sci. 29 (1989) 163.

[11] K. Ghose, G. M. Crippen, J. Chem. Inf. Comput. Sci. 27 (1987) 21.

[12] N. Bodor, Z. Gabanyi, and C. K. Wong, J. Am. Chem. Soc. 111 (1989) 3783. 
[13] A. Gavezzotti, J. Am. Chem. Soc. 105 (1983) 5220.

[14] K. J. Miller, J. Am. Chem. Soc. 112 (1990) 8533.

[15] SPSS software packages, SPSS Inc., 444 North Michigan Avenue, Suite 3000, Chicago, Illinoi, 60611, USA

[16] J. Wang, X.Q. Xie, T. Hou, X. Xu, Fast J. Phys. Chem. A. 111 (2007) 4443-4448.

[17] G. L. Patrick, An Introduction to Médicinal Chemistry, Oxford University Press, Oxford 1995, 140-141.

[18] N. I. Zhokhova, I. I. Baskin, V. A. Palyulin, A. N. Zefirov, N. S. Zefirov. Russ. Chem. $B+.52$ (2003) 1061.

[19] R. Mazri, S. Belaidi, A. Kerassa, T. Lanez, International Letters of Chemistry, Physics and Astronomy 14(2) (2014) 146-167.

[20] J. Petit, N. Maurice, C. Kaiser, G. Maggiora, Bioorg. Med. Chem. 20 (2012) 5343-5351.

[21] G. Thirunarayanan, International Letters of Chemistry, Physics and Astronomy 5 (2014) 89-98.

[22] G. Alamelumangai, N. Santhi, International Letters of Chemistry, Physics and Astronomy 5 (2014) 124-133.

[23] Dipti L. Namera, Umed C. Bhoya, International Letters of Chemistry, Physics and Astronomy 11(2) (2014) 159-166.

[24] Hasmukh R. Khunt, Piyush P. Pipaliya, Satish M. Ghelani, Jayesh S. Babariya, Yogesh T. Naliapara, International Letters of Chemistry, Physics and Astronomy 12 (2014) 20-25. 